\section{The urologist is a key determinant of ADT use in men with prostate cancer}

Whether a patient with prostate cancer receives androgen-deprivation therapy (ADT) has more to do with the supervising urologist than with patient or tumor characteristics, reports a US study.

Shahinian and colleagues obtained data from the Surveillance, Epidemiology, and EndResults and Medicare databases on men aged $\geq 66$ years, diagnosed with incident prostate cancer in 1992-1999 $(n=61,717)$, and on the urologists who treated them for 1 year postdiagnosis $(n=1,802)$. The percentages of the total variance in ADT use (within 6 months of cancer diagnosis) attributable to several variables were calculated by multilevel analyses-their first use in this setting.

The urologist was responsible for $22.56 \%$ of the variance in ADT use for men diagnosed in 1997-1999, compared with $9.71 \%$ and $4.29 \%$ for tumor and patient characteristics, respectively; although most pronounced in 1997-1999, this pattern was also evident in 1992-1996 and 1992-1999.

The authors subdivided patients diagnosed in 1997-1999 according to whether ADT use was 'evidence-based' (i.e. consistent with data published in 1997 that showed a survival advantage for ADT in locally advanced prostate cancer) or of 'uncertain benefit'. The variance attributed to the urologist was similar in both groups, strengthening earlier findings that there is substantial uncertainty about when it is appropriate to use ADT in prostate cancer. The authors acknowledge other potential influences on the urologists' decision to use ADT (including financial incentives and pressure from patients), and highlight the potential of targeting interventions (e.g. education) at urologists, to influence the way ADT is used.

Original article Shahinian VB et al. (2006) Determinants of androgen deprivation therapy use for prostate cancer: role of the urologist. J Natl Cancer Inst 98: 839-845

\section{Does cathelicidin have a role in the immune protection of the urinary tract?}

Cathelicidin, an antimicrobial peptide that kills urinary-tract pathogens in vitro, has been shown to be involved in the defense of the urinary tract against bacterial infection.

Chromek and colleagues showed that cathelicidin is constitutively expressed at low levels in human renal cortex and urothelial cells. While no intracellular peptide is usually present in these cells, exposure to bacteria causes a rapid increase in the amount of cathelicidin released, perhaps resulting from inhibition of cathelicidin mRNA degradation. The authors also observed a 'second-wave' release of cathelicidin from leukocytes, once infection was established. The murine equivalent of cathelicidin was demonstrated to exert its protective effects by limiting the attachment of bacteria to the urinary tract-an essential feature of infection.

Cathelicidin was present at markedly higher levels in urine from children with urinary tract infections than in urine from healthy children. Interestingly, strains of $E$. coli isolated from children with pyelonephritis were more resistant to cathelicidin than strains from children with cystitis, which suggests that the severity of bacterial invasion is related to resistance to cathelicidin.

Only one gene coding for cathelicidin has been found in humans; it is speculated that future studies might investigate the possibility that single-nucleotide polymorphisms in the cathelicidin gene are associated with susceptibility to recurrent or severe UTIs.

Original article Chromek M et al. (2006) The antimicrobial peptide cathelicidin protects the urinary tract against invasive bacterial infection. Nat Med 12: 636-641

\section{Immediate androgen deprivation treatment beneficial in men with aggressive prostate cancer}

It is not clear at what stage androgen deprivation treatment (ADT) should be given to patients with prostate cancer. Although ADT has good short-term efficacy in patients with symptomatic metastases, it cannot cure the disease; as a result, $A D T$ has traditionally been delayed until late in the disease course. Some studies, however, have indicated that early use of longterm ADT improves survival, and Messing et al. report further data supporting this concept.

In total, 98 men who had undergone radical prostatectomy and bilateral pelvic lymphadenectomy for node-positive, clinically localized prostate cancer were randomized to receive either immediate ADT $(n=47)$ or deferred ADT 